\title{
Risk Entropy Model of Public Security Video Surveillance System
}

\author{
Dou Wei ${ }^{1}$ Zhang Hongzhou ${ }^{1}$, ${ }^{\text {, * Zhao Xingtao }}{ }^{1}{ }^{\text {, }}$ Du Zhiguo ${ }^{1}{ }^{2}$ Li Peiyue ${ }^{1}$ \\ 1 School of Police Information Engineering and Network Security, People's Public Security University of China, \\ 2 Key Laboratory of Security Prevention Technology and Risk Assessment of the Ministry of Public Security \\ Beijing, China \\ zhanghongzhou@ppsuc.edu.cn
}

\section{公共安全视频监控系统的风险熵模型及应用}

\author{
窦伟 ${ }^{1}$ 张鸿洲 ${ }^{1,2}$ 赵兴涛 ${ }^{1,2}$ 杜治国 ${ }^{1,2}$ 李培岳 \\ 1 中国人民公安大学警务信息工程与网络安全学院 \\ 2 安全防范技术与风险评估公安部重点实验室 \\ 北京市西城区木樨地南里 1 号, 100038 \\ zhanghongzhou@ppsuc.edu.cn
}

\begin{abstract}
From the point of view of the demand of public security application for video information application, this paper compares the characteristics of traditional video surveillance system with that of urban public security video surveillance system, analyses the factors affecting the effectiveness evaluation of video surveillance system, and puts forward the evaluation index set for evaluating video information collection. On this basis, this paper establishes a risk entropy model of public security video surveillance system, and divides the risk of application of video surveillance system into the risk related to fixed target, the risk related to moving target and the risk related to image information quality satisfaction. Among them, the risk related to the moving target can be further divided into the self-risk of the target and the coupling risk between the goals, which are often related to the occurrence of the case or event. The risk entropy model of video surveillance proposed in this paper can be used to evaluate the application efficiency of video surveillance system and guide the layout of video surveillance system in a region.
\end{abstract}

Keywords-Video surveillance; risk entropy; front-end deployment; application efficiency

摘要一本文从公共安全应用对视频信息应用需求的角度出 发, 对传统视频监控系统与城市公共安全视频监控系统的特 性进行比较, 分析了影响视频监控系统效能评估的因素, 进 而提出了评价视频信息采集的评估指标集合。在此基础上, 本文建立了公共安全视频监控系统的风险摘模型, 将视频监 控系统应用的风险分为与固定目标相关的风险、运动目标相 关的风险和图像信息质量满意度相关的风险等几个部分。其 中，与运动目标相关的风险可进一步分为目标的自身风险和 目标间的耦合风险, 后者往往与案 (事) 件的发生相关。本 文提出的视频监控风险摘模型结合应用场合和应用需求的不 同配置要求, 可以用于视频监控系统应用效能的评估, 进而 指导一个区域的视频监控系统的布局。

关键词一视频监控，风险摘，系统布局，应用效能

\section{I. 引言}

视频监控能够直观反映被监控场所的实时信息, 是

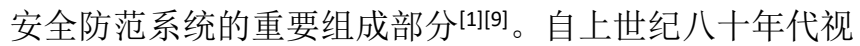
频监控被成功应用于博物馆的安全防范以来，其应用范 围不断扩大。 ${ }^{1}$ 目前, 视频监控技术在灾害监控、环境保 护、交通管理、治安管控等主要的社会公共安全应用领 域都发挥着不可替代的作用, 视频监控系统已经成为城 市公共安全的基础设施。其中, 公安业务领域内对视频 监控的应用从系统建设到应用方式都极具代表性, 特别 是在案 (事) 件侦破、治安监控、应急指挥等方面更是

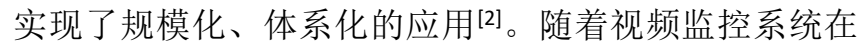
城市公共安全等领域的应用逐渐走向普及, 人们开始关 注各类视频监控系统在建设、运行和维护方面的科学合 理性, 对视频监控系统的效能的研究也成为了热点问题。

针对重点目标和场所的安全防范系统包括：入侵报 警系统、视频监控系统、出入口控制、中央控制等几个 子系统 ${ }^{[3]}$ 。通过视频监控系统, 安全保卫人员可以直观、 准确、及时地获得监控场所的信息。在安保场景中, 其 视频监控系统的前端摄像机部署在一个相对比较封闭的 内部区域, 即所谓的警戒区域或保护区域等。这些区域 有着明确的边界, 可对异常案 (事) 件, 如越界行为、

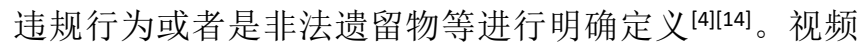
监控一般与出入口控制、报警系统联动, 主要发挥着对 报警信息的复核和对现场画面的记录作用。随着计算机 视觉 ${ }^{[5][11]}$ 等技术的进步, 目标识别侦测等技术被应用到 视频监控系统中, 使视频监控系统也具备了报警功能。 但是视频监控在安防系统中的主要作用还是没有改变。

安全防范系统的防护性能, 即安防系统的效能对实 际应用非常重要。一般来说, 系统的效能是指系统在规 定条件下完成一定任务的能力。而安防系统的有效性表 现在对人为攻击或破坏的探测、延迟、抵御等能力。因

* 通讯作者: 张鸿洲, 男, 邮箱: zhanghongzhou@ppsuc.edu.cn 
此, 可以用被保护对象受到侵害的风险来进行度量 ${ }^{[5]}$, 可表示为公式（1）：

$$
\mathrm{R}=P_{A}\left(1-P_{E}\right) \mathrm{C}
$$

上式中, $\mathrm{R}$ 表示系统的安全风险, $\mathrm{C}$ 表示系统被攻 击或破坏后的严重程度, $P_{A}$ 表示在一定时间内系统被攻 击的可能性。因此, $\left(1-P_{E}\right)$ 可以理解为安全防范系 统面对一定威胁的脆弱性, $P_{A}$ 和 $\left(1-P_{E}\right)$ 的乘积是成 功攻击的概率。在传统的安全防范系统中，视频监控可 以通过提高复核效率来降低 $\left(1-P_{E}\right)$ 的值来提高整个 系统的安全防护效能。

近年来，视频监控系统的应用环境从室内扩大到城 市公共开放空间, 从应用方式、应用目的等方面都有了 的新变化，呈现出新的特性。

与传统的作为安防系统的一部分的视频监控系统不 同，城市视频监控系统被部署在开放的环境中，虽然可 以人为的划定一些区域并进行重点监控, 但是与安防系 统的禁区或控制区域有所不同。这些区域仍然属于公众 可以自由出入的公共区域, 只是出于安全管理等目的, 需要相关安保人员加以留意。因此, 城市视频监控系统 很难, 甚至不可能去定义什么是非法越界、异常案 (事) 件和异常行为。此外, 开放环境下的成像条件会随着时 间的变化而变化。例如, 摄像机部署地点的周围景观会 随时间产生变化, 并且光照条件也会随时间的变化产生 复杂变化。这些都给成像带来了巨大的挑战, 严重地影 响了摄像机采集信息的质量, 进而给后续的应用造成了 很大的困难。

公共安全领域内对于视频监控系统应用的目的与传 统安全防范系统中的视频监控有很大的不同。以城市视 频监控系统为例, 它主要被应用于公安部门, 使用者是 警察，并且应用方式也由对报警信号进行复核检查转变 为用于支撑决策的信息来源。在（城市）公共开放环境 下, 视频监控是一个视觉信息传感网络, 它能够记录自 然条件以及社会生活的影像资料, 即监控视频是社会生 活和自然条件的视觉镜像。对于公安部门而言, 其应用 目的是利用视频监控系统解决一些业务, 例如利用视频 监控在案 (事) 件发生前起到预警与安全防范的作用; 在案 (事) 件发生后可以起到支撑公安业务及决策的作 用。本质上来说, 社会公共安全领域中的视频监控, 主 要是为各类公安全事务的决策提供信息支撑，而不是直 接用于打击犯罪 ${ }^{[17]}$, 因此对它的效能评价不能直接使用 公式 (1) 来表示, 需要重新构建风险的度量模型。

从视频监控系统的构成及每一个部分的功能来看, 它的效能问题可以分为硬件系统的可靠性分析、前端数 据采集的效能、数据处理的效率与准确性、应用功能的 实用性等多个方面。除摄像机外, 视频监控系统的硬件 的绝大部分属于典型的 IT 设备 ${ }^{[7]}$, 而对于这类设备的可 靠性研究已经非常成熟, 可以直接将其引入到视频监控 系统硬件设备的效能评估中。从视频监控对公共安全决 策所需的信息要求的支撑程度来看, 视频信息的数量和 质量能否满足实际需要决定着决策的正确与否: 充分可 靠的信息支撑能够最大可能的减少由于盲目决策带来的 安全风险。基于上述考虑并仿照公式（1）的原则，对 于一个需要监控的场所或一定范围内的目标, 将视频监 控应用的风险表示为:

$$
\mathrm{R}=\mathrm{P}\left(1-\mathrm{P}^{\prime}\right) \mathrm{W}
$$

上式中, 因子 $\mathrm{P}$ 表示监控目标出现在监控场所的概 率, $\mathrm{P}^{\prime}$ 表示前端摄像机能够采集满足要求的信息的概率。 相应的, $\left(1-\mathrm{P}^{\prime}\right)$ 表示监控系统采集到的信息不能满 足要求的概率, 这种情况下系统就无法为后续的应用提 供必要的信息支持。 $\mathrm{W}$ 是目标的视觉信息对于满足决策 需求的权重。在不同的应用场景以及要求中, $\mathrm{W}$ 的值将 会有所不同。显然, 权重 $\mathrm{W}$ 的取值与视频监控工作的重 要性或者监控目标发生社会公共安全事件所带来的后果 有关。

根据上述对监控应用风险定义, 接下来本文将从公 共安全视频监控系统信息采集出发, 首先分析影响效能 的因素, 然后从应用需求的角度出发, 提出评价视频信 息采集的评估指标集。最后, 利用指标集合建立公共安 全视频监控系统的风险熵模型。

\section{II. 视频监控系统信息采集的要求及影响因素}

视频监控系统可以看作一个传感网络, 传感器是监 控摄像机。信息传感的对象主要是人及其社会活动, 而 人的活动受一定的空间分布特征影响。具体来说, 城市 空间及其之上的社会单位分布决定了人的社会生活行为 特征, 而人的社会生活更进一步地决定了公共安全问题 的特征。考察社会公共安全领域视频监控系统的效能问 题, 可以从分析城市空间的地理结构对视频监控系统的 信息采集单元的影响入手。

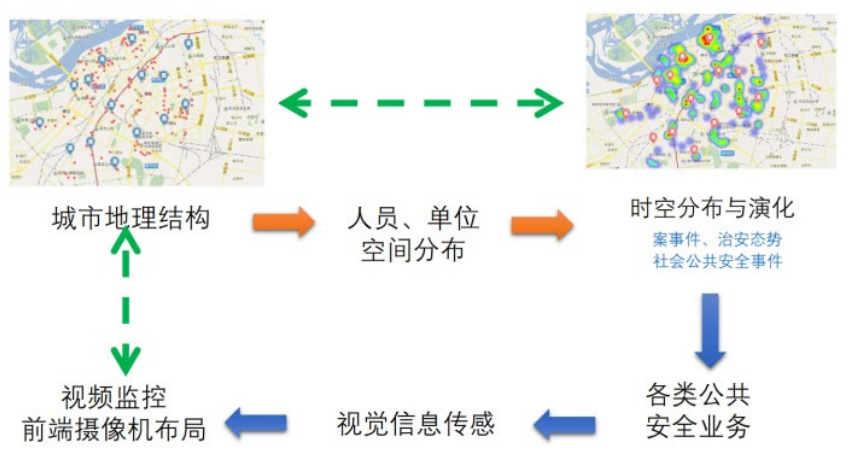

图 1. 考虑地理因素的监控系统布局逻辑示意图

环境心理学的研究表明, 自然环境的空间分布特征 会及大地影响到人们的行为模式。此外, 犯罪学的相关 研究 ${ }^{[8]}$ 也表明, 街头犯罪的时空分布特征在城市的空间 分布呈现出一定的规律性。综合两方面的研究, 可以得 出这样的结论: 城市空间地理结构决定了社会单位的空 间分布或布局以及人员在一定范围内的流动模式, 这其 中也蕴藏了犯罪行为发生的时间和空间分布。因此, 视 频监控系统应该在空间中，从社会公共安全的信息采集 要求出发进行布局, 其逻辑关系如图 1 所示。因此, 城 市视频监控系统前端摄像机需要通过一定的方式实现对 空间关键点的信息采集覆盖。从布局结构上看, 还需要 与城市地理结构特征、地理信息分布特征相适应。公安 视频监控系统在建设过程中总结出了 “圈、块、线、点” [9]这一基本的前端摄像机布防结构。即城市及其功能区 外围利用监控摄像机构成防控圈、块封闭的信息采集结 构; 在目标通行路径沿途关键部位部署摄像机来构建视 频监控信息采集的线状结构; 在重要单位周边或部位处 部署摄像机来构建视频监控防控点等。按照 “圈、块、 
线、点”布防结构, 视频监控系统形成了网格化、多层 次立体的视频信息采集形态。

在防灾减灾、环境保护等其他公共安全领域的问题, 也具有上述类似的特征。例如, 围绕可能会对社会生产 生活产生重大影响的灾害易发的地区构建圈、点结合的 视频监控点。但是，人迹罕至的地区的地质事故一般不 纳入公共安全检测的目标范围，也不需要对其进行视频 监控 ${ }^{[10]}$

此外，视频监控信息采集的种类和数量要求应当针 对不同的应用需求，建立相应的评估指标。以公安业务 涉及的视频监控为例，治安管理、案（事）件侦查等虽 然存在一些视频监控信息需求的共性, 但是不同类型业 务的信息需求差别较大。治安管理需要对一定空间范围 内目标 (主要是人员) 的活动情况进行监视, 以判断是 否存在非法聚集等异常活动或其他影响公共安全的事件 [11]，因此摄像机画面能够尽可能全面地反映监控区域是 监控任务的首要需求, 即要求摄像机拍摄的覆盖面积要

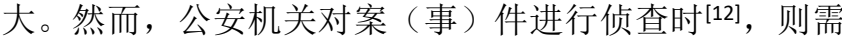
要摄像机画面尽可能地提供目标的细节信息, 以便检测 和识别目标的身份属性。因此, 监视画面能否反映目标 的主要特征区域，如人脸部分或体貌特征是视频监控的 首要要求。当然, 治安管理也期望获得目标的细节特征, 案 (事) 件侦查同样希望获得目标在一个较大范围内的 运动轨迹信息。但是这些视频信息的需求优先级相对较 低，并且在具体细节上有所不同。

总之，环境因素对于视频监控目标的空间分布特征 及其行为特征的影响至关重要。一方面, 由于摄像机被 部署在一定的自然环境和社会环境中，环境因素影响着 信息采集的过程。另一方面，视频信息的采集不能脱离 具体的应用需求，不同类型的应用对视频信息的种类、 数量等存在不同的要求（或者说对其的重要程度不同）。 因此, 视频监控系统的建设, 需要对环境因素着重考虑。

\section{III. 视频监控风险熵}

熵是物理学和信息科学中的重要概念, 是对系统混乱 程度的度量 ${ }^{[14]}$ 。传统的安防系统由若干防护节点组成, 在每一个节点上均可以部署多个传感器, 对系统受到的 攻击行为进行全面探测, 因此安防系统被看作是由具有 确定探测率的传感器构成的信息通信系统。文献[15]提 出了基于节点防护满意度的风险熵模型，该模型将安防 系统的效能评估问题将转化为 Neyman-Pearson 准则下的 优化问题。社会公共安全领域内视频监控系统的效能体 现在能否获得相关安全应用所需的视频信息。如果视频 监控系统提供的信息能够满足应用的决策判断需求，则 能够有效降低相关的安全风险。由此可见, 复杂的地理 空间结构以及目标的行为活动对社会公共安全应用所需 的信息有一定影响，具体的量化计算必然是一个非常复 杂的过程。幸运的是，公共安全的具体应用对视频信息 的需求往往是明确给出的。例如, 公共安全应用中识别 目标所需的视频信息可以用目标区域的像素数表示 ${ }^{[13]}$ 。 因此，本文将文献[15]定义的风险熵扩展到公共安全视 频监控系统:

$$
\mathrm{S}=-\sum \omega_{\mathrm{i}} \log \mathrm{R}_{\mathrm{i}}
$$

式中， $\mathrm{R}_{\mathrm{i}}$ 是采集的视觉信息满足应用需求的程度，其 值越大表明越能满足应用需求，其对于决策的不确定性
就越小。在等式 (2) 中, $\mathrm{P}^{\prime}$ 由摄像机的覆盖类型以及部 署细节有关, $\mathrm{R}_{\mathrm{i}}$ 是基正是于此进行计算的, 下标 $\mathrm{i}$ 表示不 同类型的视觉信息需求。 $\omega_{i}$ 是对应于 $R_{i}$ 的第 $i$ 个指标的权 重。对照公式 (2) 不难发现其物理意义: 公共安全领 域内的监控目标发生安全事件的概率 $\mathrm{P}$, 其本质上是由 环境因素所决定的; $\mathrm{P}^{\prime}$ 是获取到期望的视频信息的概率, 主要由摄像机的部署细节所决定, 但也受环境中的成像 条件等因素所影响; 以一定概率获得的某种视频信息的 权重 W 则具体由具体的应用需求来决定。

公共安全应用中的风险描述往往是针对监控目标进行 表述的。这些典型的目标包括具有历史和政治意义的广 场、高价值物品、人员以及典型的事件等。

\section{（1）与固定目标或区域相关的风险熵}

给定一个视频监控任务, 需要对固定目标 $\mathrm{T}_{\mathrm{i}}(\mathrm{i}=1,2, \ldots$ $n)$ 和控制区域 $A_{j}(j=1,2, \ldots m)$ 进行监控。固定目标被看作 一个点, 其监视要求可以用一定的覆盖程度来表示, 只 有未被任何相机覆盖的目标才会有固定目标的风险熵。 对于一级覆盖请求, 每个固定目标 $\mathrm{T}_{\mathrm{i}}$ 的风险商为:

$$
\mathrm{S}_{1, \mathrm{i}}=-\log \frac{\sum \mathrm{R}_{\mathrm{s}, \mathrm{k}} \mathrm{p}_{\mathrm{k}}}{\sum \mathrm{R}_{\mathrm{s}, \mathrm{i}} \mathrm{p}_{\mathrm{i}}} \quad\left(\mathrm{R}_{\mathrm{s}, \mathrm{i}} \neq 0, \mathrm{p}_{\mathrm{i}} \neq 0\right)
$$

式中, $p_{i}$ 是 $T_{i}$ 卷入安全事故的概率，例如，某个危险 物品爆炸的概率为 $\mathrm{p}_{\mathrm{i}}, \mathrm{R}_{\mathrm{s}, \mathrm{i}}$ 表明在发生公共安全事件 时, $\mathrm{T}_{\mathrm{i}}$ 造成的影响, 或者说 $\mathrm{T}_{\mathrm{i}}$ 的重要性, 表下标 $\mathrm{K}$ 表示 $\mathrm{T}_{\mathrm{i}}$ 中被视频监控覆盖的目标。

类似地，控制区域 $\mathrm{A}_{\mathrm{j}}$ 产生的风险熵可以用下式表示:

$$
\mathrm{S}_{1, \mathrm{j}}=-\log \frac{\sum \mathrm{R}_{\mathrm{w}, \mathrm{j}} \mathrm{p}_{\mathrm{j}} \mathrm{a}_{\mathrm{j}}}{\sum \mathrm{R}_{\mathrm{w}, \mathrm{j}} \mathrm{p}_{\mathrm{j}} \mathrm{A}_{\mathrm{j}}}\left(\mathrm{R}_{\mathrm{w}, \mathrm{j}} \neq 0, \mathrm{p}_{\mathrm{j}} \neq 0, \mathrm{~A}_{\mathrm{j}} \neq 0\right)
$$

式中, $\mathrm{a}_{\mathrm{j}}$ 是监控覆盖的第 $\mathrm{j}$ 个控制区域的大小， $A_{\mathrm{j}}$ 是 整个区域的大小, 用 $a_{\mathrm{j}} / \mathrm{A}_{\mathrm{j}}$ 表示区域的覆盖率。 $\mathrm{p}_{\mathrm{j}}$ 是在区 域 $A_{j}$ 发生安全案（事）件的概率。 $R_{w, j}$ 与 $R_{S, i}$ 类似, 表示 控制区域的重要性。如果 $a_{\mathrm{j}} / \mathrm{A}_{\mathrm{j}}=1$, 则方程式将退化为 (4)。

综上，与固定目标和控制区域有关的视频监控风险商 为前两部分的加权和:

$$
\mathrm{S}_{1}=\omega_{\mathrm{i}} \mathrm{S}_{1, \mathrm{i}}+\omega_{\mathrm{j}} \mathrm{S}_{1, \mathrm{j}}
$$

（2）与移动目标相关的风险熵

将移动目标所带来的视觉监视风险熵 $\mathrm{S}_{2}$ 进一步又分为 $\mathrm{S}^{\mathrm{M}}$ 和 $\mathrm{S}^{\mathrm{MS}}$ 两个部分, $\mathrm{S}^{\mathrm{M}}$ 的值完全是由移动目标 (如非 法聚集的人群或车辆）决定:

$$
S^{M}=-R_{w} \log \left(P \mid P_{M}\right)
$$

式中, $\mathrm{P}_{\mathrm{M}}$ 是发生公共安全事件的概率, $R_{w}$ 表示其重 要性, 则 $\left(P \mid P_{M}\right)$ 是在发生安全事件的情况下, 拍摄到移 动目标的条件概率, 这种概率可以用车辆的例子来解 释。忽略嫌疑人的反侦查意识或视频监控对人的安全感 的影响, 则 $\mathrm{p}$ 和 $\mathrm{P}_{\mathrm{M}}$ 之间没有相互作用, 是相互独立的, 则上式变形为:

$$
S^{M}=-R_{M}\left(\log P+\log P_{M}\right)
$$

$S^{M S}$ 描述了移动目标与固定目标之间相互作用的不确 定性，与 $\mathrm{S}^{\mathrm{M}}$ 类似，忽略了摄像机部署的影响： 


$$
\begin{aligned}
S^{M S} & =-R_{M S} \log \left(P \mid P_{M S}\right) \\
& =-R_{M S}\left(\log P+\log P_{M S}\right)
\end{aligned}
$$

在以上讨论中，如果有多个移动目标与其他目标发生 交互作用, 下标 $\mathrm{q}$ 就应该附在每个变量上。总风险摘 $S_{2}$ 应该与所有目标都有关,即计算他们的和:

$$
S_{2}=\sum_{q}\left(S_{q}^{M}+S_{q}^{M S}\right)
$$

（3）与视觉信息质量相关的风险熵

对图像质量的要求包括图像尺寸、清晰度、对比度、 色彩精度等。假设有一系列指标 $\mathrm{D}_{\mathrm{k}}, \mathrm{k}=1,2, \ldots$, 一个特定 任务的图像质量要求可以用每个指标的满意度 $\theta_{\mathrm{k}}$ 及其相 应的权重 $\omega_{\mathrm{k}}$ 来表示, 则由视觉信息质量进一步决定的决 策不确定性为:

$$
\begin{aligned}
S_{V Q} & =-\sum_{K} \omega_{K} \log \left(\theta_{K} \mid P\right) \\
& =-\sum_{K}\left(\log \theta_{K}+\log P\right)
\end{aligned}
$$

式中, $p$ 是摄像机捕捉到目标的概率, $\theta_{\mathrm{k}}$ 可视为随机 变量; 一般情况下, 图像质量与概率 $\mathrm{p}$ 无关, 条件概率 $\left(\theta_{\mathrm{k}} \mid \mathrm{p}\right)$ 等于概率 $\mathrm{p}$ 和满意度 $\theta_{\mathrm{k}}$ 的乘积。 和:

综上，总的视频监控风险熵等于以上三部分的加权

$$
\mathrm{S}=\alpha S_{1}+\beta S_{2}+\gamma S_{3}
$$

式中, $\mathrm{S}_{1}$ 和 $\mathrm{S}_{2}$ 表明在给定的警用视频监控任务中 受关注的目标的类型, 权重因子 $\alpha$ 和 $\beta$ 反映了它们的重要 性; 对于 $\mathrm{S}_{\mathrm{VQ}}$ 和因子 $\gamma$, 它们表达了图像细节在任务中的 重要性。对于一个确定的视频监控任务, 权重因子的配 置是唯一的, 例如, 监视任务只是集中在移动目标上, 那么描述控制区域和固定目标重要性的权重将被设为零。

\section{IV. 视频监控风险熵的应用}

在上面的内容中，本文提出了一个视频监控系统的 风险熵模型, 下面以云台变焦相机（Pan/Tilt/Zoom, PTZ）的视场优化为例进行模型的具体用途说明。

PTZ 相机是一种具有调节监控角度和调焦能力的监 控相机, 其视场角是描述摄像机覆盖的一个关键指标, 由方位角 $\theta, \Phi$ 和焦距 $\mathrm{f}$ 决定。使用 PTZ 相机对监控区 域进行监控时, 需要对其控制参数进行预设。而以往的 控制参数设定都是根据经验或者是个人喜好，并且其合 理性无法给出保证。可以利用本文的风险熵模型优选出 满足一定要求的监视范围。这就要求视频监控摄像机信 息采集时的风险熵为最小值:

$$
\arg \min \left\{\alpha S_{1}+\beta S_{2}+\gamma S_{3}\right\}
$$

根据前面的（2）式，场所中发生安全事件的概率 $\mathrm{P}^{\prime}$ 一般是确定的, 是一个大于零的常数。同时, 监控目 标的行为模式从统计学角度来看也是确定的, 但是其空 间的分布形态未知。此外，空间不同位置处监控目标的 出现概率 P 可以借助于目标统计技术, 如行人检测算法 得到。因此, 要达到式 (13) 的优化目标就需要对具体 的需求进行量化表示。文献[18]针对一个室内的监控场 景下的固定监控目标和监控区域, 在考虑到对场所中的 行人外貌视频采集要求的情况下, 采用前述模型进行了 优化计算。如图 2 所示, 左图给出了两个固定目标 $D_{1}$ 和
$D_{2}$, 两个限制区域 $A_{1}$ 和 $A_{2}$ 的示意图, 右图给出了优化结 果。红色虚线框 $M_{1} 、 M_{2}$ 、和 $M_{3}$ 为熵值总和最小的前三 个视场。根据这三个视场范围和成像的几何关系可以推 算出 PTZ 相机的控制参数并进行相应的设置。

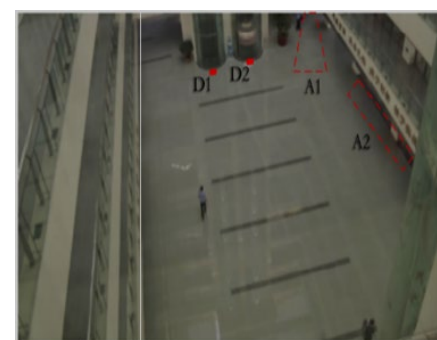

(a) 最广视图及监控目标

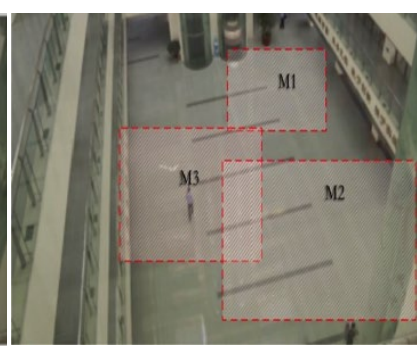

（b）优化结果区域
图 2. 监视区域优化的实例 ${ }^{[18]}$

在上面应用的基础上还可以进一步用于协调多个摄 像机间的监控任务, 此类应用方式在公共安全视频监控 中通常被称为协同防控 ${ }^{[16]}$ 。针对协同防控的优化过程在 计算风险熵时，需要考虑多个视角之间的信息冗余问题， 这涉及到对多个摄像机风险熵的求和问题。城市范围的 视频监控布局需要考虑犯罪活动的空间分布特点, 运用 本文提出的风险商模型时, 需要将 “圈、块、线、点” 的城市布防结构需求转化为相应的覆盖指标, 并且结合 POI 的分布与覆盖率之间的关系做进一步的分析。

在具体的监控点位布设问题中, $\mathrm{P}$ 代表的是目标的 出现在摄像机中的概率, 在宏观布局中, 认为视频监控 点位均部署在合理的位置, 此时可以假设监控目标出现 并被监控摄像机拍到的概率是一样的。再对区域内 POI 密度与监控点位密度的关系进行分析, 结合覆盖要求得 到合理的监控布局。将实际的布局情况与之进行比较, 可以发现监控薄弱区域, 进而可以做出及时的调整。

\section{V. 结论与讨论}

迄今为止, 已有大量针对安全防范效能评估的论述研 究, 但是将开放公共环境中的视频监控系统作为独立系 统进行考察, 采用定量建模的方式对视频监控运用中的 风险问题进行研究是一个新问题。本文提出的模型对视 频监控应用中经常遇到的 “看不清” 和 “看不见” 的问 题进行了有效描述。所谓的 “看不清” 除了指设备性能 问题外, 主要还是由于摄像机视场范围过大带来图像目 标区域过小、目标细节不清晰的问题; “看不见” 问题 通常是指前端建设不足以及摄像机的覆盖盲区问题。公 式 (12) 的第一、二部分主要考虑如何尽量多的将监控 区域和监控目标纳入到摄像机视场中, 也就是对视频目 标信息的数量的度量; 而第三部分重点考虑到对视频目 标信息质量的度量, 可用的指标如目标区域的像素数。 显然, 要保证视频目标信息的质量, 就不能无节制的扩 大摄像机的视场覆盖, 所以本文所提出的模型要求能够 在优化过程中, 保证采集到的视频信息要尽可能的满足 实际应用需求, 克服 “看不清” 问题。模型考虑到了实 际应用对视频信息数量和质量两方面的要求, 可以在摄 像机视场优化中找到熵最小的平衡点。此外, 由于本文 定义的熵模型是从实际应用的角度出发, 基于对应用需 求的符合度进行建模, 这避免了对大量参数的计算。

针对本文所提出的问题, 进一步研究除了前面提到的 用于解决多摄像机协同监控和城域范围的布局优化问题 
外, 还需要针对公共安全应用, 如公安业务, 对视频信 息的需求进行量化建模。实际应用对于视频覆盖的要求, 特别是公式 (11) 所描述的视频监控信息质量的需求, 往往是定性的描述, 并且不同的业务所需求的重点也各 有不同。因此，建立一整套有关视频信息数量和质量的 指标体系及其应用的标准化参数体系是应用本文模型的 关键前提之一。此外, 如何在数学上通过进一步标准化 需求参数以保证熵的基本数学特性, 也是进一步工作需 要重点研究的问题。

\section{致谢}

本研究得到国家重点研发计划课题（编号: 2016YFC0801003）和公安部基础工作专项项目（编号： 2016GABJC01）资助。

\section{参考文献}

[1] 黄凯奇, 陈晓棠, 康运锋, 谭铁牛. 智能视频监控技术综述 [J]. 计算 机 学报, 2015,38(6).

[2] Zhang Zhengrong.Talking about the construction and management of public security video surveillance system.China Public Security, 2013 (13) : 130-133.

[3] Garcia, M. L., In Vulnerability Assessment of Physical Protection Systems. Elsevier Butterworth-Heinemann: Oxford, UK, 2006, ISBN: 978-0-7506-7788-2.

[4] Cocca, P.; Marciano, F.; Alberti, M., Video surveillance systems to enhance occupational safety: A case study. Safety Science 2016, 84 140-148, DOI: 10.1016/j.ssci.2015.12.005.

[5] Tsakanikas, V.; Dagiuklas, T., Video surveillance systems-curren status and future trends. Computers \& Electrical Engineering 2017, DOI: 10.1016/j.compeleceng.2017.11.011.

[6] 赵兴涛、张翔、陈志华. 安全防范系统脆弱性评估 [M]. 中国人民 公安大学出版社: 北京市, 2015 .
[7] Amac Guvensan, M., Gokhan Yavuz, A., On coverage issues in directional sensor networks: A survey, Ad Hoc Networks 2012, 9(7), 1238-1255, DOI: 10.1016/j.adhoc.2011.02.003.

[8] Cheng, T.; Williams, D., Space-time analysis of crime patterns in central London. 2012; Vol. 39.

[9] Chen Rongbo, Luo Yong, Xie Wenhai.Discussion on selection of public security monitoring point.Modern Business Trade Industry, 2014: (1) : 197-198.

[10] Piciarelli C, Foresti G L.PTZ network configuration for optimal 3Dcoverage.IEEE International Conference on Advanced Video\&amp;Signal Based Surveillance, 2011:435-437.

[11] Chen Gang, Xu Lei.On the building of work mechanism of video investigation.Journal of Shandong Police College, 2012; (9) :53-57.

[12] Chen Gang, Xu Lei.Research on image detection method of video surveillance.Journal of Chinese People's Public Security University (Social Sciences Edition), 2012; (3) :120-125.

[13] International Electrotechnical Commission, Video surveillance systems for use in security applications In Part 4: Application guidelines, 2014, ISBN: 978-2-8322-1504-3.

[14] 胡瑞敏, 吕海涛, 陈军. 基于风险熵和 Neyman-Pearson 准则的安防 网络[J].自动化学报, 2014,40(12).

[15] Hu, R.; Lv, H.; Chen, J., Risk Evaluation Model of Security and Protection Network Based on Risk Entropy and Neyman-Pearson Criterion. Acta Automatica Sinica 2014, 40 (12), 2737-2746, DOI: 10.3724/SP.J.1004.2014.02737.

[16] Wang X. Intelligent multi-camera video surveillance: A review. Pattern Recognition Letters, 2012 34(1):3-19.

[17] The Effective of Public Space CCTV: A Review of Recent Published Evidence Regarding the Imapct of CCTV on crime. Police and Community Safety Directorate Scottish Government. 2009.

[18] Hongzhou Zhang, Zhiguo Du, Xingtao Zhao, Peiyue Li, and Dehua Li "A risk-based coverage model for video surveillance camera control optimization", Proc. SPIE 9815, MIPPR 2015: Remote Sensing Image Processing, Geographic Information Systems, and Other Applications, 98151B (14 December 2015); 